\title{
BMJ Open Perceptions of drones, digital adherence monitoring technologies and educational videos for tuberculosis control in remote Madagascar: a mixed- method study protocol
}

\author{
Elysée Nouvet, ${ }^{1}$ Astrid M Knoblauch, ${ }^{2,3}$ Ian Passe, ${ }^{4}$ Andry Andriamiadanarivo, ${ }^{5}$ \\ Manualdo Ravelona, ${ }^{5}$ Faniry Ainanomena Ramtariharisoa, ${ }^{5}$ \\ Kimmerling Razafimdriana, ${ }^{5}$ Patricia C Wright, ${ }^{4,5}$ Jesse McKinney, ${ }^{4,5}$ \\ Peter M Small, ${ }^{4}$ Niaina Rakotosamimanana, ${ }^{3}$ Simon Grandjean Lapierre ${ }^{\oplus 3,6,7}$
}

To cite: Nouvet $\mathrm{E}$ Knoblauch AM, Passe I, et al. Perceptions of drones, digital adherence monitoring technologies and educational videos for tuberculosis control in remote Madagascar: a mixed-method study protocol. BMJ Open 2019;9:e028073. doi:10.1136/ bmjopen-2018-028073

- Prepublication history and additional material for this paper are available online. To view these files, please visit the journal online (http://dx.doi. org/10.1136/bmjopen-2018028073).

Received 20 November 2018 Revised 22 February 2019 Accepted 4 March 2019

D) Check for updates

(c) Author(s) (or their employer(s)) 2019. Re-use permitted under CC BY-NC. No commercial re-use. See rights and permissions. Published by BMJ.

For numbered affiliations see end of article.

\section{Correspondence to}

Simon Grandjean Lapierre; simon.grandjean.lapierre@ umontreal.ca

\author{
ABSTRACT \\ Introduction Poor road and communication infrastructure \\ pose major challenges to tuberculosis (TB) control in many \\ regions of the world. TB surveillance and patient support \\ often fall to community health workers (CHWs) who may \\ lack the time or knowledge needed for this work. To \\ meet the End TB Strategy goal of reducing TB incidence \\ by $90 \%$ by 2035 , the WHO calls for intensified research \\ and innovation including the rapid uptake of new tools, \\ interventions and strategies. Technologies that 'leapfrog' \\ infrastructure challenges and support CHWs in TB control \\ responsibilities have the potential to dramatically change \\ TB outcomes in remote regions. Such technologies may \\ strengthen TB control activities within challenged national \\ tuberculosis treatment and control programmes (NTPS), \\ and be adapted to address other public health challenges. \\ The deployment of innovative technologies needs to \\ be differentially adapted to context-specific factors. \\ The Drone Observed Therapy System (DrOTS) project \\ was launched in Madagascar in 2017 and integrates a \\ bundle of innovative technologies including drones, digital \\ adherence monitoring technology and mobile device- \\ based educational videos to support TB control. \\ Methods and analysis This mixed-methods study \\ gathers and analyses cultural perceptions of the \\ DrOTS project among key stakeholders: patients, \\ community members, CHWs, village chiefs and NTP_ \\ DrOTS mobile health teams. Data from questionnaires, \\ semistructured interviews, focus group discussions \\ (FGD) and ethnographic observation gathered from \\ June 2018 to June 2019 are thematically analysed and \\ compared to identify patterns and singularities in how \\ DrOTS stakeholders perceive and interact with DrOTS \\ technologies, its enrolment processes, objectives and \\ team. \\ Ethics and dissemination Ethics approval was obtained \\ from the National Bioethics Research Committee of \\ Madagascar and Stony Brook University institutional \\ review board. Study results will be submitted for peer- \\ reviewed publication. In Madagascar, results will be \\ presented in person to Ministry and other Malagasy
}

Strengths and limitations of this study

This study is one of the first to assess acceptance and perceptions of drones and evriMED pillboxes (medication reminder and digital remote treatment adherence monitoring devices), technologies currently being considered for scale-up in several health systems around the world. The bundle of technologies at the core of this study is one that could be applied in response to other health needs in other contexts.

- Qualitative methods facilitate detailed and nuanced understanding of how and why stakeholders with limited literacy and in remote settings perceive and use new technologies.

- Data are collected from a range of stakeholders and focused on those using these new technologies on the front-lines in low-income countries (ie, patients, national mobile healthcare team members, community health workers and villagers).

- Findings from perception studies serve to deepen understanding of how contextual particularities can impact on acceptance, perceptions of and interactions with new technologies but may not be generalisable across distinct settings and populations.

decision-makers through the Institut Pasteur de Madagascar.

Patient and public involvement This study is designed to foreground the voices of patients and potential patients in the DrOTS programme. CHW participants in this study also supported the design of study information sessions and recruitment strategies. One member of the mobile health team provided detailed input on the wording and content of FGD and interview guides. Study findings will be presented via a report in French and Malagasy to $\mathrm{CHW}$, mobile health team and other village-level participants who have email/internet access. 


\section{INTRODUCTION}

Ten million people developed active tuberculosis (TB) disease in 2017. ${ }^{1}$ To meet the End TB Strategy goal of reducing incidence by $90 \%$ and mortality by $95 \%$ before 2035, the WHO has called for intensified research and innovation including the rapid uptake of new tools, interventions and strategies. ${ }^{2}$ In low-income and middle-income countries (LMIC) that are disproportionately more affected by disease, TB control can be hindered by any number of social and structural factors, including limited access to centralised facilities for populations living outside the capital, understaffed healthcare infrastructures, poor development of laboratory diagnostic networks or stigma influencing healthcare seeking behaviours and treatment adherence. ${ }^{34}$ In such contexts, diagnosis may be delayed or fail to occur at all, and implementation of TB control essential strategies such as case finding, access to laboratory diagnosis, directly observed therapy and contact tracing by national tuberculosis control programmes (NTPs) becomes particularly challenging, increasing secondary transmission and fatalities. ${ }^{5}$

In 2017, nearly $30000 \mathrm{~TB}$ cases were reported in Madagascar. ${ }^{6}$ This corresponds to half of actual cases to have occurred in the country, as estimated by WHO. ${ }^{6}$ Treatment adherence and success rates are reported to be highly variable between regions of the country, respectively, averaging $60 \%$ and $84 \%$ in the Drone Observed Therapy System (DrOTS) intervention area and at national level. ${ }^{67}$ Madagascar exemplifies the challenges of quality TB care delivery for remote and dispersed populations as $40 \%$ of Malagasy people live more than $5 \mathrm{~km}$ from the nearest basic healthcare facility, with no public transit system or even roads in many cases. ${ }^{8} \mathrm{~TB}$ diagnosis and treatment challenges in Madagascar are representative of those in many areas of sub-Saharan Africa: (i) underserved healthcare system; (ii) poverty and cultural norms hindering healthcare seeking; (iii) paucity of human resource capacities with training in TB; (iv) paucity of diagnostic facilities; (v) suboptimal coverage of treatment and treatment follow-up and (vi) high prevalence of two important TB risk factors, that is, malnutrition and indoor air pollution. ${ }^{5910}$ Conversely, factors of good prognosis for TB control in Madagascar include very low rates of both multi-drug resistant TB (MDR-TB) infection and HIV-TB coinfection in the country. ${ }^{611}$

To leapfrog over theses impediments to quality TB care, the DrOTS project was coinitiated with the Malagasy NTP and was deployed by Stony Brook University's Global Health Institute in collaboration with the Institut Pasteur de Madagascar. The project was implemented in 61 randomly selected villages in Androrangavola commune, south-eastern Madagascar, in August 2017. Up to $70 \%$ of the approximately 27000 inhabitants living in Androrangavola commune live more than $5 \mathrm{~km}$ away from the closest healthcare facility, and that facility in turn is situated more than 1 day's walk from the closest TB diagnosis centre. ${ }^{12}$ DrOTS involves an NTP-DrOTS mobile health team consisting of TB nurses and doctors who collaborate with local community health workers (CHWs). DrOTS implements active case finding and supports TB screening, diagnosis, counselling and treatment supervision within communities using a suite of technologies: drones, evriMED pillboxes and tablet-based educational videos. In this scheme, drones increase access to diagnosis and care by facilitating specimen transport and securing the medication supply chain between diagnosis and treatment centres and remote communities; the evriMED supports TB treatment adherence and monitoring by beeping when patients need to take their medication, and tracking for healthcare providers when the evriMED has been opened (equated with dose being taken); and a tablet-based eHealth video curriculum supports patients and CHWs by providing key information on TB care and prevention at every step of the pathway to cure. Given the novelty of this approach, we designed and presented here a study protocol for the DrOTS perception study, a mixed-method cultural acceptability substudy embedded within the DrOTS project.

Several organisations are exploring the economic and practical feasibility of using drones for healthcare purposes including support to rescue missions in disasters; enhanced epidemiological monitoring for disease outbreaks and vectors; delivery of critical resources such as blood or defibrillators in emergencies; delivery of other routine and occasional medical payloads such as samples for laboratory analysis, vaccines, medication and supplies for community healthcare centres. ${ }^{13-18}$ Medication reminder and remote treatment adherence monitoring devices, such as the evriMED pillbox, may augment treatment adherence and facilitate more effective allocation of limited healthcare personnel resources in contexts where populations are hard to reach, or health systems under-resourced. ${ }^{19-21}$ While the potential of new digital technologies to transform healthcare is enormous, this potential is contingent on user-specific and context-specific needs, engagements with technologies and digital health strategies, and may imply different impacts and challenges in different contexts. ${ }^{22}$

This study will shed light on cultural and individual perceptions, barriers and facilitators to implementation of the unique bundle of technologies that make up the DrOTS system. To facilitate the implementation of such innovative technologies, other challenges such as sustained funding, creation of an LMIC-friendly market environment for drone supplies and aviation regulation approval also need to be overcome: such barriers are not addressed here.

\section{Rationale}

Determining the success of the DrOTS project cannot be limited to evaluation of its impacts on additional TB notifications or completed treatments. Though such measures are central to establishing the value and potential of innovative technologies in the global fight against $\mathrm{TB}$, the success of innovative public health strategies is also contingent on how those on the receiving end of 
these perceive and engage with its objectives, enrolment, participation processes and technologies. The DrOTS perception study aims to build expertise on how to implement new technologies in a way that is acceptable to individuals and communities that lack close or affordable access to TB diagnosis and treatment, have no prior exposure to such technologies and may harbour understandings of TB that limit management of presumptive or confirmed infections. Attending to these contextual and lived particularities of the DrOTS project is key to designing and planning feasible and effective scale-up. Recently published WHO guidelines will certainly help TB control programmes choosing and implementing digital adherences monitoring technologies. ${ }^{22}$ At present, there exists no ethical or practical guidance on the contextually sensitive use of such technologies or for the use of drones for disease diagnosis and treatment. This study can inform needed evidence-based guidance for the future expansion of such technology suites poised to strengthen TB programmes and healthcare systems more generally.

\section{Primary objectives}

1. To identify prevalent perceptions (eg, perceived benefits, concerns, misunderstandings) related to the various technological and programmatic aspects of the DrOTS project at the level of TB patients, members of patient households, other village members, CHWs, village chiefs and NTP-DrOTS mobile health team.

2. To generate a description of how and why individuals, families and villages are interacting with specific components of the DrOTS project in unanticipated ways.

\section{Secondary objectives}

1. To establish rates and demographic distribution of understandings of and interactions with the various technological and programmatic aspects of the DrOTS project.

2. To generate understandings of how and based on what factors individuals, families and villages are deciding whether or not to participate in the DrOTS project.

3 . To develop a set of evidence-based cultural and contextual considerations that can inform the implementation of similar technology-mediated diagnostic and treatment in other regions of Madagascar and in other national contexts.

\section{METHODS AND ANALYSIS \\ Study design}

The DrOTS perception study is a mixed-methods study involving cross-sectional data collection through (i) questionnaires ( 750), (ii) focus group discussions FGDs ( 19), (iii) semistructured in-depth interviews $(\sim 24)$ and (iv) ethnographic observation (figure 1). Quantitative questionnaire data are collected throughout the DrOTS project period (November 2017-December 2018) as well as an additional 6-month after project end for follow-up of TB patients adhering to a 6-month treatment regimen. Data collection for the qualitative part is initiated in June 2018 with a first village visit, followed by second and third village in August and November 2018, respectively. Ethnographic observation occurs between June and July 2018. All study tools were developed by content experts in dialogue with members of the DrOTS

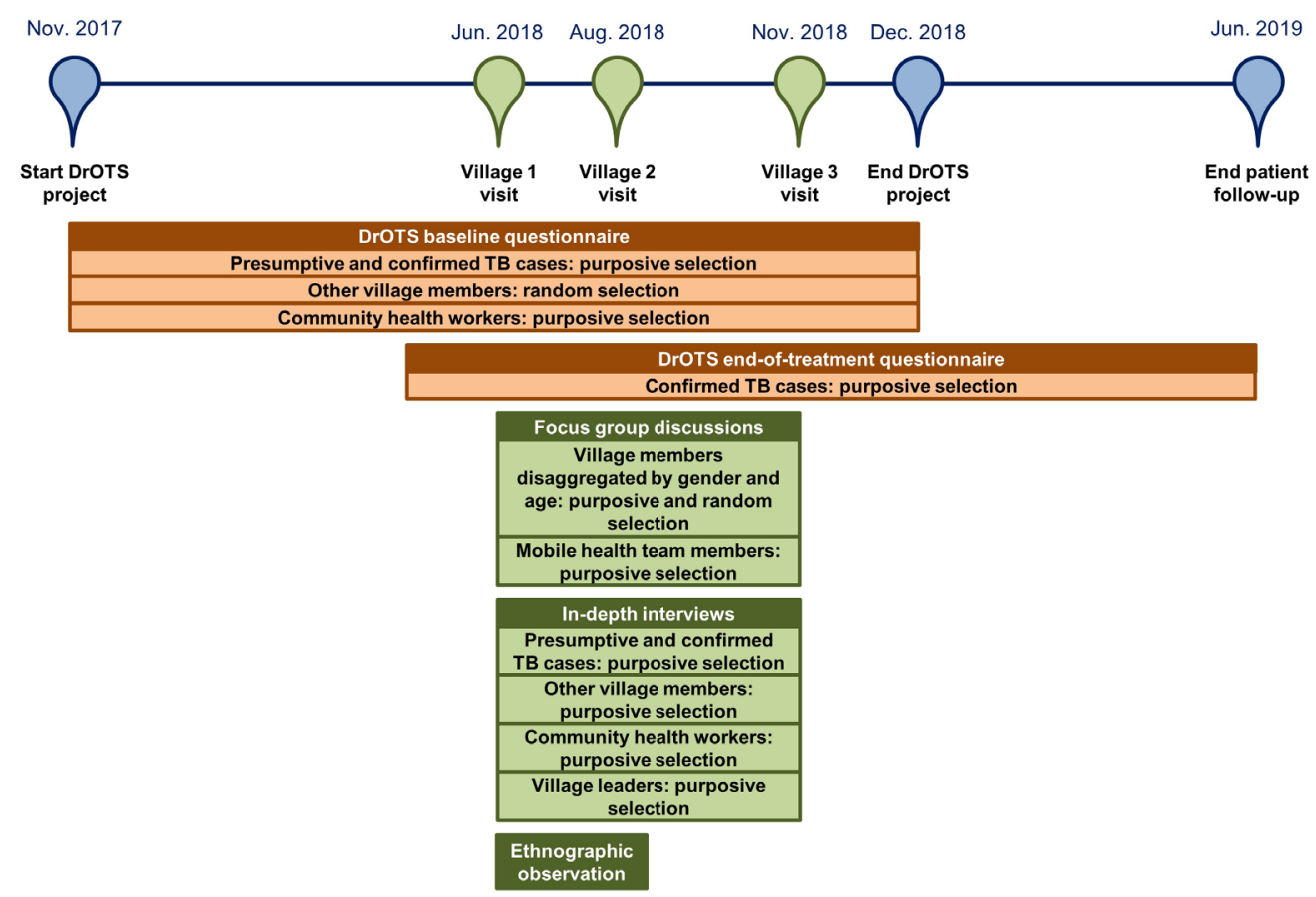

Figure 1 Timelines, approaches and tools, DrOTS perception study, Madagascar, 2017-2019. DrOTS, Drone Observed Therapy System; TB, tuberculosis. 
team responsible for community engagement and/or familiar with local dialect and customs in Androrangavola commune.

\section{Questionnaire survey}

The use of the questionnaire survey in this mixed-methods study aims to support measurement and comparison of key acceptability indicators between different study subgroups (eg, patients vs non-patients, most educated vs least educated). The questionnaire is being administered to different groups including patients, members of patient households, other village members, CHWs, village chiefs and NTP-DrOTS mobile health teams.

Two questionnaires are being used: (i) DrOTS baseline questionnaire conducted with presumptive and confirmed TB cases, other village members and CHWs (see online supplementary material 1-DrOTS Perception Study Baseline Questionnaire), and (ii) DrOTS end-of-treatment questionnaire conducted with confirmed TB cases (see online supplementary material 2-DrOTS Perception Study End-of-Treatment Questionnaire). Questionnaires are administered verbally by members of the NTP-DrOTS mobile health team to 10-15 individuals aged $\geq 15$ years in each of the DrOTS-participating villages, amounting to a total of around 750 completed baseline questionnaires. The sample size was calculated based on (i) a total population size of 27000 in Androrangavola commune, (ii) on the assumption that $50 \%$ reply 'Yes' to one main question on drone perception (ie, 'Do you think that the drones bring something positive to your community?', (iii) a 95\% confidence level and (iv) a $10 \%$ drop-out rate. This resulted in a sample size of 417 . However, the overall DrOTS-project design, lifespan and geographic coverage allowed us to sample a larger population. All self-presenting presumptive (including later confirmed) TB cases and CHWs are purposively invited to answer the baseline questionnaire, with remaining participants being randomly recruited in each DrOTS village. This questionnaire provides quantitative data on basic sociodemographic characteristics, travel history, TB risk factors, TB-related health seeking behaviour, medical history, knowledge on TB and behaviours towards TB. An end-of-treatment questionnaire is administered to all enrolled TB patients on completion of treatment. This questionnaire addresses specific perceptions of drones, evriMED and educational videos. Questionnaire data are collected using tablet-based Open Data Kit (ODK) software standardised form.

\section{Focus group discussions}

FGD-based qualitative data collection is taking place in three of the 61 DrOTS participating villages as well as with the NTP-DrOTS mobile health team. Villages for the running of FGDs are selected based on ${ }^{1}$ participation in the DrOTS project for at least 3 months $^{2}$; accessibility for the qualitative research team, meaning within a day's hike from the closest vehicle-accessible town ${ }^{3}$ and willingness to host the DrOTS perception team. Collecting data in three villages enables comparison of reported perceptions and acceptability of new technologies across villages, the possibility for diversity in perceptions to be captured. Furthermore, even where similarities exist, a more nuanced understanding of how village-specific characteristics, such as access to livelihood activities, norms of postsecondary education achievement and historical relations to outsiders travelling in the region (eg, for mining or development initiatives) may shape perceptions of the DrOTS project.

Sampling of FGD participants is purposive and randomised. It is purposive inasmuch as we are aiming to gather perceptions from a range of individuals who have engaged with DrOTS first-hand. For FGDs, we also aim to have an equal representation of men and women, and an equal representation across age groups in villages, towards capturing potential diversity in experiences of DrOTS based on diverse levels or types of responsibility in the home and village. Villagers willing to partake in a FGD provide their names to the research team, and may or may not be randomly selected to join the FGD organised for their age and gender group. Randomised selection of village FGD participants avoids burdening CHWs or other leaders with the task of identifying potential participants (which could also potentially result in biased responses tied to particular village interests). Also, in our experience, many are interested in participating in FGDs, and this randomised selection ensures all those interested have and know they have an equal chance of being selected (names of volunteers drawn from hat at village meeting).

Homosociality and age-group separation are dominant norms guiding social interaction in this part of Madagascar. Hence, to facilitate participants feeling at ease and to limit the possibility of social hierarchies in a FGD over-determining who feels able to express their view in a group, FGDs are being organised along age and gender lines: married men, married women, unmarried men, unmarried women, elder men and elder women (table 1). FGDs are run using a standard set of questions organised into two parts. Part I focuses on deepening the understanding of the participants' day-to-day definition and management of illness and understandings of TB prior to DrOTS and part II focuses on perceptions and interactions with DrOTS. The set of questions for the NTP-DrOTS mobile health team FGD is distinct, and probes members' understanding and concerns related to DrOTS, as well as perceptions of cultural and village-specific attitudes, knowledge and engagements with specific aspects of DrOTS pilot project based on work in all villages (see online supplementary material 3-DrOTS Perception Study FGD Guide). All FGDs are moderated and/or supervised by an experienced medical anthropologist and conducted in Malagasy local dialect with the help of a translator. With participants' permission, these are digitally recorded. 


\begin{tabular}{|c|c|c|c|}
\hline Location & Stakeholder group & $\begin{array}{l}\text { Target } \\
\text { no } \\
\text { groups }\end{array}$ & $\begin{array}{l}\text { Participants/ } \\
\text { group }\end{array}$ \\
\hline \multirow[t]{6}{*}{ Village 1} & Unmarried women & 1 & 4 \\
\hline & Unmarried men & 1 & 4 \\
\hline & Married women & 1 & 4 \\
\hline & Married men & 1 & 4 \\
\hline & Elderly women & 1 & 4 \\
\hline & Elderly men & 1 & 4 \\
\hline \multirow[t]{6}{*}{ Village 2} & Unmarried women & 1 & 4 \\
\hline & Unmarried men & 1 & 4 \\
\hline & Married women & 1 & 4 \\
\hline & Married men & 1 & 4 \\
\hline & Elderly women & 1 & 4 \\
\hline & Elderly men & 1 & 4 \\
\hline \multirow[t]{6}{*}{ Village 3} & Unmarried women & 1 & 4 \\
\hline & Unmarried men & 1 & 4 \\
\hline & Married women & 1 & 4 \\
\hline & Married men & 1 & 4 \\
\hline & Elderly women & 1 & 4 \\
\hline & Elderly men & 1 & 4 \\
\hline $\begin{array}{l}\text { National TB } \\
\text { Programme }\end{array}$ & $\begin{array}{l}\text { Mobile health unit } \\
\text { team members }\end{array}$ & 1 & 5 \\
\hline Total & & 19 & 77 \\
\hline
\end{tabular}

DrOTS, Drone Observed Therapy System; TB, tuberculosis.

\section{Semistructured in-depth interviews}

Semistructured in-depth interviews gather information on different stakeholders' knowledge of, attitudes towards, uses and first-hand experiences of the DrOTS project that cannot be gleaned from questionnaires, and may be too sensitive or detailed to emerge in FGDs (online supplementary material 4-DrOTS Perception Study SemiStructured Interview Guide). Recruitment is purposive and targets five categories of key stakeholders in each village 1) DrOTS project-enrolled adult patients (over 15 years of age) 2) adults who presented for TB testing to the DrOTS team but have received negative diagnostic testing results 3) villagers hesitant, unable or have declined to join the project 4) CHWs 5) village leaders, including kings and elected district chiefs. All participants except for participants from category 3 are being identified with help from the NTP-DrOTS mobile team. Participants in category 3 are invited to self-identify in the process of FGDs and may be identified by other interview participants using 'snowball sampling'. An estimated eight interviews will be performed in the same subset of three villages as FGDs for a total of 24 interviews (table 2). Additional interviews are performed with TB
Table 2 Semistructured interview recruitment plan, DrOTS perception study, Madagascar, 2017-2019

\begin{tabular}{lll}
\hline Location & Stakeholder group & Participants \\
\hline Sample village & DrOTS enrolled patient & 2 \\
& $\begin{array}{l}\text { Villagers who tested } \\
\text { negative for TB }\end{array}$ & 2 \\
& $\begin{array}{l}\text { Villagers hesitant, unable or } \\
\text { who have declined DrOTS } \\
\text { participation }\end{array}$ & 2 \\
& Community health worker & 1 \\
& Village leaders & 1 \\
Total & & 8 \\
\hline
\end{tabular}

DrOTS, Drone Observed Therapy System; TB, tuberculosis.

confirmed cases outside the included villages to increase the number of DrOTS-enrolled patients.

\section{Ethnographic observation}

An anthropology trainee will seek permission to stay with one DrOTS patient in their village over a 2-week period in order to better understand, through ethnographic observation, how that individual lives with TB disease on a daily basis and interacts with DrOTS. The trainee will keep a record of what they notice and learn (field notes), attending to 1 ) ways in which patients, villagers, village leaders, the CHW in the village, or any members of the mobile health team present in this 2-week period discuss DrOTS with the patient or one another, 2) technical, practical community or individual level challenges, 3) any differences or similarities in attitudes or beliefs about the DrOTS pilot project study expressed in informal conversation about the project and/or its technologies and 4) contextualised information about how the patient at the centre of this observation views and uses DrOTS technologies. Ethnographic observation often occurs over a period of months or even years; however, even shorter applications of this method can build contextual understanding of healthcare projects. Use of this method in this study may confirm or reveal gaps in the information gathered through the other data collection methods, and serve to identify questions for future research.

\section{Analysis}

Questionnaire quantitative data will be descriptively analysed using STATA V.14.0 (StataCorp; Texas, USA) and will address and compare levels of acceptability between groups.

Interviews and FGDs will be transcribed and translated into English by a professional Malagasy translator mastering the field study site local dialect. Transcripts will be uploaded into Nvivo V.12.0 (QSR; Melbourne, Australia) and be subjected to thematic analysis. Categories of enquiry in interview and FGD guides will form the basis for an initial coding structure. Three semistructured interviews will then be independently coded by two investigators to test and adjust this structure in light of the data, 
adding themes to account for unanticipated but relevant content. In an iterative process, minor adjustments and additions (eg, change in theme names or merging of themes) to the codebook will be made when needed. Key theme and subtheme contents will be summarised and reviewed to allow linkage of quantitative and qualitative data, clearly identify themes with exemplary quotes, raise questions and concerns, and inform guidelines for culturally and contextually sensitive technology implementations. Ethnographic field notes will not be coded but will inform the analysis by providing support to findings from the interviews and FGDs, and/or by identifying gaps knowledge gaps.

\section{DISSEMINATION}

Following project and technology sensitisation visits and in accordance with cultural norms, consent is obtained from the local leaders to present this research project to villagers under their responsibility before engaging in any activities within villages. Though limited literacy is widespread in this region of Madagascar, as per national research ethics norm, consent forms are read and explained to participants and written informed consent is obtained from any and all parties agreeing to participate before conducting interviews, focus groups and observations. Data are being anonymised rendering participants' identification from dissemination material impossible. Participants in this perceptions study do not face any different healthcare as a result of participation. All patients diagnosed with TB within the DrOTS projects are treated for free in accordance with NTP and WHO guidelines.

One issue that requires managing in such a study is the risk of social stigma for participants. TB and association with TB are stigmatised in contexts around the world, and well documented in sub-Saharan Africa (eg, ${ }^{3423-25}$ While there is no evidence of TB stigma in the Malagasy context of our research, we are adopting strategies to mitigate the risk of TB-related stigma developing for participants in our TB-focused study. In our initial meeting with CHWs in each village, we are stressing our interest in speaking with DrOTS-enrolled patients (TB active) but also our commitment to keeping the TB-active status of these patients private. We will be working with CHWs in villages to identify strategies to protect the TB-active status of any patients we interview. In presenting the study to the entire village, we are stressing our interest in understanding the villagers' - and not just presumptive or confirmed TB positive individuals'-perceptions of the DrOTS programme. The number of individuals, including respected elders, and range of visible healthiness of those who are participating in either an interview or FGD in each village does reduce the possibility of a single individual among a village's participants being associated with $\mathrm{TB}$. We will not carry out the ethnographic observation component of this study if doing so risks rendering public a currently private TB diagnosis.
Results of the DrOTS perception study will be submitted for peer-review publication. A two-page summary of results will be prepared in French and Malagasy and included as an appendix to the results paper, and shared with participants and collaborators for whom we have contact information. In Madagascar, results will be presented in person to Ministry and other Malagasy decision-makers through the Institut Pasteur de Madagascar.

\section{DISCUSSION}

This study responds to a current gap in knowledge on the feasibility and cultural acceptability of using a new suite of technologies including evriMED, drones and tablet-mediated video education to support improved TB diagnosis and treatment in remote populations. Digital technologies that enable remote monitoring and faster delivery of medical care and supplies are potential game changers for healthcare landscapes struggling with long-standing or temporary (eg, due to disasters) barriers to healthcare delivery. Still, the development of innovative healthcare technologies does not guarantee their enthusiastic and rapid adoption in diverse settings. ${ }^{26-28}$ Perceived benefits and risks, use and challenges of adopting such innovation is not likely to be uniform across distinct sociocultural, health system and economic settings. These may vary depending on any number of factors, including (to name just a few) the presence or lack of alternative options for care, association of use with a context-specific stigmatised condition, as well as prior direct or rumoured experiences with interventions deemed to be similar in some way to the new ones. There is inherent ethical complexity in using devices such as drones for 'surgical strikes' on 'wicked' global health challenges, if key determinants of poor health remain unaddressed. ${ }^{29}$ Recent studies conducted with intended users and 'beneficiaries' of new digital health systems in LMIC contexts reveal other context-specific and culture-specific concerns or limitations of these technologies, such as concerns these could dehumanise assistance, ${ }^{28}$ infringe on private spaces ${ }^{30}$ and be too expensive for some governments. ${ }^{30}$ Findings from the DrOTS cultural acceptability study may or may not reveal similar concerns in the Malagasy context, and will be situated within growing literature on perceptions and acceptability of TB remote digital medical monitoring and adherence strategies.

While focused on one project in Madagascar, the DrOTS perception study provides a model for attending to contextual factors that may affect target population support and intended interactions with any number of other new public health initiatives around the world. Embedding such perception studies within projects is particularly important for initiatives conceptualised based on the theoretical but as of yet unproven potential of new technologies. While findings from perceptions studies on new innovative technology-mediated health projects may not be readily generalisable, as each setting is unique, such studies can generate learning that is transferable to 
other settings by drawing attention to the range of factors that may impact on technology adoption and interaction, and by shedding light on how others on the front line of innovative projects have sensitively navigated the cultural, social, political and ethical complexities of particular contexts. In these ways, perception studies that 'localise' understandings of how theoretically promising new technologies work in specific locales have an important role to play in informing evidence-based guidance for context-sensitive implementation and scale-up of programmes such as DrOTS around the world.

\section{Limitations and potential challenges}

The significance of results from this study will be difficult to ascertain given limited research on drone supported TB or other public health programmes at this juncture. Future research on similar programmes introduced in Madagascar or elsewhere will be helpful in assessing the generalisability of our eventual results to other regions and populations where such technologies for increased healthcare provision are being introduced. This study does not include interviews or FGDs with National TB programme policy-makers and decision-makers. Understanding rationales underlying government approval of pilot programmes such as DrOTS among individuals working for national TB control in the country, as well as the challenges and perception of outcomes and impact among these stakeholders constitute equally important research as we work towards clarifying what it means to develop context-appropriate use of innovative technologies in TB control.

Respondents may overemphasise positive perceptions of DrOTS. Social desirability bias is a challenge in perceptions studies, especially where there may be unspoken assumption among participants that their responses to study questions could negatively impact future programmes. ${ }^{31}$ Our work across multiple villages will increase our ability to detect and probe overly positive accounts from participants. We are confident in the study's design and its ability to generate accurate and detailed insight into perceptions of DrOTS; however, we recognise the possibility that with a focus on only three of the 61 DrOTS-enrolled villages, our qualitative data collection may not capture the full range of perceptions that may be present among Malagasy villagers enrolled in DrOTS.

\section{Author affiliations \\ ${ }^{1}$ School of Health Studies, Western University, London, Ontario, Canada ${ }^{2}$ Epidemiology and Public Health, Swiss Tropical and Public Health Institute, Basel, Basel-Stadt, Switzerland \\ ${ }^{3}$ Mycobacteria Unit, Institut Pasteur de Madagascar, Antananarivo, Madagascar ${ }^{4}$ Global Health Institute, Stony Brook University, Stony Brook, New York, NY, USA ${ }^{5}$ Health Department, Center Valbio, Ranomafana, Madagascar \\ ${ }^{6}$ Immunopathology, Centre de recherche du CHUM, Montreal, Quebec, Canada ${ }^{7}$ Microbiology, Infectious Diseases and Immunology, Université de Montréal, Montréal, Québec, Canada}

Acknowledgements We are grateful to the DrOTS project Malagasy team including community healthcare workers involved in patient and data management. We also thank the Madagascar's National Tuberculosis Control Program personnel for their collaboration, reviewers Kevin Schwartzman and Dennis Falzon for their careful review and feedback on this protocol paper, and Dr Mamy Fiononana Ratofotera Raelison for his feedback on the study tools.

Contributors All authors meet criteria for authorship as per the International Committee of Medical Journal Editors recommendations. EN, AMK, IP, NR, PMS $A A$ and SGL designed study protocol and data collection tools. EN, IP, AA, FAR, MR, JM, NR and SGL are involved in field implementation and data collection. EN, AMK, IP, AA, PCW, PMS and SGL are involved in data management and interpretation. All authors have read and approved the final version of the manuscript before submission.

Funding The DrOTS project was supported by the Stop TB Partnership's TB REACH initiative and was funded by the Government of Canada. IP receives financial support from the Stony Brook University Undergraduate Research and Creative Activities Award program. AMK is supported by the Rudolf Geigy Foundation, Swiss Tropical and Public Health Institute, Basel, Switzerland. EN receives financial support from the Western University Faculty Development Fund Grant program. SGL is supported by the Canadian Association for Microbiology and Infectious Diseases.

Competing interests Authors declare no financial or personal relationships with other people or organisations that could inappropriately influence this work. The authors have no relationship and obtained no funding for this study from the developers of evriMEDTM. The first and corresponding author had full access to all the data in the study and had final responsibility for the decision to submit this work for publication.

Patient consent for publication Not required.

Ethics approval This study received institutional review board approval from both the 'Comité d'Éthique de la Recherche Biomédicale' from the Ministry of Public Health in Madagascar, Madagascar (073-MSANP/CERBM) and Stony Brook University, New York, USA (CORIHS\# 2017-4056F).

Provenance and peer review Not commissioned; externally peer reviewed.

Open access This is an open access article distributed in accordance with the Creative Commons Attribution Non Commercial (CC BY-NC 4.0) license, which permits others to distribute, remix, adapt, build upon this work non-commercially, and license their derivative works on different terms, provided the original work is properly cited, appropriate credit is given, any changes made indicated, and the use is non-commercial. See: http://creativecommons.org/licenses/by-nc/4.0/.

\section{REFERENCES}

1. WHO. Global TB Report. 2018.

2. WHO. WHO end TB strategy. Geneva, Switzerland: WHO, 2016.

3. Chimbatata NBW, Zhou CM, Chimbatata CM, et al. Post-2015, why delay to seek healthcare? Perceptions and field experiences from TB healthcare providers in northern Malawi: a qualitative study. Infect Dis Poverty 2017;6:60.

4. Cramm JM, Finkenflügel HJ, Møller V, et al. TB treatment initiation and adherence in a South African community influenced more by perceptions than by knowledge of tuberculosis. BMC Public Health 2010;10:72.

5. Yates TA, Khan PY, Knight GM, et al. The transmission of Mycobacterium tuberculosis in high burden settings. Lancet Infect Dis 2016;16:227-38.

6. WHO. Country Tuberculosis Profile - Madagascar. 2017.

7. MSANP, DGS, DLT. Plan stratégique national de lutte contre la tuberculose à Madagascar 2015-2019. Antananarivo: Ministère de la Santé Publique, Direction Générale de la Santé, Direction de Luttre contre la Tuberculose, 2015.

8. Ratovonirina NH, Rakotosamimanana N, Razafimahatratra SL, et al. Assessment of tuberculosis spatial hotspot areas in Antananarivo, Madagascar, by combining spatial analysis and genotyping. BMC Infect Dis 2017;17:562.

9. Barmania S. Madagascar's health challenges. Lancet 2015;386:729-30.

10. Instat I. Enquête démographique et de santé 2008-2009. Antananarivo: Institut National de la Statistique and ICF Macro, 2010.

11. UNAIDS. HIV Country Profile - Madagascar 2016. 2016.

12. Miller AC, Ramananjato RH, Garchitorena A, et al. Baseline population health conditions ahead of a health system strengthening program in rural Madagascar. Glob Health Action 2017;10:1329961.

13. Claesson A, Fredman D, Svensson L, et al. Unmanned aerial vehicles (drones) in out-of-hospital-cardiac-arrest. Scand J Trauma Resusc Emerg Med 2016;24:124.

14. Harnett BM, Doarn CR, Rosen J, et al. Evaluation of unmanned airborne vehicles and mobile robotic telesurgery in an extreme environment. Telemedicine journal and e-health 2008;2008:539-44. 
15. Katariya M, Chung DCK, Minife T, et al. Drone inflight mixing of biochemical samples. Anal Biochem 2018;545:1-3.

16. McCall B. Sub-Saharan Africa leads the way in medical drones. The Lancet 2019;393:17-18.

17. Frontières MS. Innovating to reach remote TB patients and improve access to treatment, MSF. 2014 https://www.msf.org/papua-newguinea-innovating-reach-remote-tb-patients-and-improve-accesstreatment (Accessed 2 Oct 2017).

18. UNICEF. Vanuatu announces drone trial participants to assess vaccine delivery in remote islands:UNICEF. $2017 \mathrm{https}: / /$ reliefweb.int/ report/vanuatu/vanuatu-announces-drone-trial-participants-assessvaccine-delivery-remote-islands (Accessed 2 Oct 2017).

19. Broomhead S, Mars M. Retrospective return on investment analysis of an electronic treatment adherence device piloted in the Northern Cape Province. Telemed J E Health 2012;18:24-31.

20. Liu X, Lewis JJ, Zhang $\mathrm{H}$, et al. Effectiveness of electronic reminders to improve medication adherence in tuberculosis patients: a clusterrandomised trial. PLoS Med 2015;12:e1001876.

21. Subbaraman R, de Mondesert L, Musiimenta A, et al. Digital adherence technologies for the management of tuberculosis therapy: mapping the landscape and research priorities. BMJ Glob Health 2018;3:e001018.

22. WHO. Handbook for the use of digital technologies to support tuberculosis medication adherence. 2018.

23. Chikovore J, Hart G, Kumwenda M, et al. TB and HIV stigma compounded by threatened masculinity: implications for TB healthcare seeking in Malawi. Int J Tuberc Lung Dis 2017;21:26-33.
24. Amo-Adjei J. Individual, household and community level factors associated with keeping tuberculosis status secret in Ghana. BMC Public Health 2016;16:1196.

25. Deribew A, Abebe G, Apers L, et al. Prejudice and misconceptions about tuberculosis and HIV in rural and urban communities in Ethiopia: a challenge for the TB/HIV control program. BMC Public Health 2010;10:400.

26. Cazabon D, Suresh A, Oghor C, et al. Implementation of Xpert MTB/RIF in 22 high tuberculosis burden countries: are we making progress? Eur Respir J 2017;50:1700918.

27. Campbell Jl, Eyal N, Musiimenta A, et al. Ethical questions in medical electronic adherence monitoring. $J$ Gen Intern Med 2016;31:338-42.

28. Soesilo D, Sandvik K. Drones in Humanitarian Action - A survey on perceptions and applications. Geneva: The Swiss Foundation for Mine Action (FSD), 2016.

29. Peckham R, Sinha R. Anarchitectures of health: Futures for the biomedical drone. Glob Public Health 2018;15:1-16.

30. Eichleay M, Mercer S, Murashani J, et al. Using unmanned aerial vehicles for development: perspectives from Citizens and Government Officials in Tanzania. Durham, USA: ICT Works, 2016.

31. Nouvet E, Abu-Sada C, de Laat S, et al. Opportunities, limits and challenges of perceptions studies for humanitarian contexts. Canadian Journal of Development Studies / Revue canadienne d'études du développement 2016;37:358-77. 